\title{
The Plant Microbiome at Work
}

\author{
Klaus Schlaeppi ${ }^{1}$ and Davide Bulgarelli ${ }^{2}$ \\ ${ }^{1}$ Plant-Soil-Interactions, Institute for Sustainability Sciences, Agroscope, Reckenholzstrasse 191, CH-8046 Zürich, Switzerland; \\ ${ }^{2}$ Division of Plant Sciences, College of Life Sciences, University of Dundee at The James Hutton Institute, Invergowrie, \\ Dundee, Scotland DD2 5DA, U.K.
}

Submitted 22 October 2014. Accepted 11 December 2014.

\begin{abstract}
Plants host distinct microbial communities on and inside their tissues designated the plant microbiota. Microbial community profiling enabled the description of the phylogenetic structure of the plant microbiota to an unprecedented depth, whereas functional insights are largely derived from experiments using individual microorganisms. The binary interplay between isolated members of the plant microbiota and host plants ranges from mutualistic to commensalistic and pathogenic relationships. However, how entire microbial communities capable of executing both growth-promoting and growth-compromising activities interfere with plant fitness remains largely unknown. Ultimately, unravelling the net result of microbial activities encoded in the extended plant genome- the plant microbiome-will be key to understanding and exploiting the full yield potential of a crop plant. In this perspective, we summarize first achievements of plant-microbiome research, we discuss future research directions, and we provide ideas for the translation of basic science to application to capitalize on the plant microbiome at work.
\end{abstract}

The plant microbiota.

The advent of massive parallel sequencing presented a step change for the field of plant-microbe interactions, because it facilitated the investigations of whole plant-associated microbial communities. The ideas that plants are holobionts (i.e., host and associated microbes) and that the associated microbes affect plant fitness were not new, but the technical advance promoted their research. This development enhanced the interest in the plant microbiota (Box 1) as testified by many recent reviews (Bakker et al. 2013; Berendsen et al. 2012; Berg et al. 2014; Bulgarelli et al. 2013; Guttman et al. 2014; Knief 2014; Lebeis 2014; Philippot et al. 2013; Porras-Alfaro and Bayman 2011; Schlaeppi et al. 2013; Turner et al. 2013). Amplicon-based community profiling approaches (Box 1) provide insights on community structure and phylogenetic diversity of the plantassociated microbes and are suitable to examine the major environmental and biological factors responsible for shaping its composition (Bulgarelli et al. 2013). However, phylogenetic profiles do not provide direct functional information of a given microbial community, i.e., the functional potential of the plant-

Corresponding authors: K. Schlaeppi;

E-mail: klaus.schlaeppi@agroscope.admin.ch and

D. Bulgarelli; E-mail: d.bulgarelli@dundee.ac.uk

(C) 2015 The American Phytopathological Society associated microbiota — the plant microbiome (Box 1)—remains largely unknown. Further developments on sequencing and computational approaches are now driving plant-microbiota investigations to plant-microbiome research focusing on the functional examination of the plant-associated microbial communities.

Deciphering the plant microbiome.

Interactions between members of the plant microbiota and their hosts cover a spectrum ranging from mutualistic to commensalistic and parasitic relationships (Box 1). Numerous studies conducted on microorganisms that were isolated from the phyllosphere, rhizosphere, leaf or root endosphere have revealed a series of microbial traits, which are beneficial from the perspective of the host plant (Lugtenberg and Kamilova 2009; Porras-Alfaro and Bayman 2011; Vorholt 2012). In particular, enhanced nutrient acquisition from soil, tolerance to abiotic stresses and hormone production, as well as indirect pathogen protection have been proposed as primary microbial mechanisms that permit the promotion of plant growth. These microorganisms are collectively referred to as PGPM (Box 1). Recent advances in plant-microbiota investigations clearly support the idea that PGPM represent particular members of the plant microbiota. However, our knowledge on PGPM is still limited to studies conducted on individual isolates under laboratory conditions (Bulgarelli et al. 2013), and we have a limited understanding how entire microbial communities contribute to plant growth. A multitude of questions arise. Do the microbial traits remain beneficial for plants when the respective microbe resides in a community context? Or, how are such plant-beneficial traits repartitioned among members of a microbiota? Do most members or individuals serve the host plant with a particular trait? What is the net outcome from the interaction of a host plant with a complex microbiota comprising members with positive, neutral, and negative activities? It is conceivable that beneficial traits are readily interchanged via HGT (Box 1) among microbiota members and, therefore, such traits rather present a community attribute. To advance our understanding of the plant microbiome, we discuss in the following section some of the holistic approaches adopted to investigate functions of the plant-associated microbial communities and we illustrate how the combination of cultivation-independent and -dependent techniques might shape plant-microbiome studies in the next years.

The meta-omics approaches.

Metagenomics was defined as the simultaneous characterization of complex DNA mixtures retrieved from a microbial habitat (Riesenfeld et al. 2004; Box 1). Metagenomics laid the 
foundations for a series of approaches investigating RNA (metatranscriptomics), proteins (metaproteomics), and metabolites (meta-metabolomics), all providing novel insights on the metabolic capacity of a given microbiota. Delmotte and co-workers (2009) used combined metaproteogenomics in which the metagenomic information assisted the annotation of the metaproteomic data. The study revealed the composition of the phyllosphere microbiota together with the expressed microbial traits in this habitat. The authors investigated the phyllosphere of clover, soybean, and Arabidopsis, and this investigation highlighted that, despite the approximately 130 million years of evolutionary divergence between Fabaceae and Brassicaceae families (Hyung et al. 2014), approximately $70 \%$ of the phyllosphere metaproteome was conserved. This finding suggests the existence of a large core microbiome with minor host-specific functions of the microbiota. A few years later, the metagenome of a root endosphere habitat was reported, based on bacterial cells retrieved from surface-sterilized rice roots, providing insights into the metabolic capacity of the microbial community inside plant roots (Sessitsch et al. 2012). By combining metagenomics with the transcriptional investigation of selected microbial genes, the authors demonstrated that bacterial microbiota inside plant roots have the potential to execute key steps of the nitrogen cycle in planta. In the same year, the microbiota and their traits were compared between the phyllosphere and rhizosphere habitats of paddy field-grown rice plants by Knief and colleagues (2012), employing metaproteogenomics. This investigation revealed habitat-specific microbial traits such as, for example, the biological fixation of nitrogen in the rhizosphere, as evidenced by the presence of di-nitrogenase reductase genes and proteins in this habitat. Recently, metatranscriptomics has been used to reveal the putative contribution of microbial communities to phytoremediation in the willow rhizosphere, representing one of the first examples of this type of investigation in plant science (Yergeau et al. 2014). These few examples clearly illustrate the major advantage of meta-omics approaches, the possibility to identify the microbial traits existent in a plant microbiota without cultivating their members.

Some limitations are intrinsically associated to the metaomics approaches. Limitations arise due to the complexity of the plant microbiota and their inherent traits (Bulgarelli et al. 2013) and because plant-associated microbiota specimens are almost invariably "contaminated" with host DNA, RNA, or proteins. Such contamination can be particularly marked in endosphere preparations as, for example, up to $90 \%$ of plant sequences were obtained from DNA preparations retrieved from Arabidopsis root samples (D. Bulgarelli, E. Ver Loren van Themaat, and P. Schulze-Lefert unpublished results). Therefore, deciphering the plant microbiome requires large sequencing coverage and, in conjunction with the need of replicated experiments for robust analyses, the financial and computational burden becomes substantial (Knight et al. 2012). Further, low rates in high-confidence annotation hamper the functional interpretation of microbial DNA, RNA, and protein sequence data. Currently, only a fraction of the sequences retrieved from a nonhuman environment, usually up to $50 \%$, can be functionally annotated with today's database coverage (Thomas et al. 2012). Ultimately, it is fair to underscore that meta investigations provide first observations or indirect correlations and additional experiments (discussed below) are needed to firmly reveal whether the observed diversification of the plant microbiome is a cause or a consequence of an observed plant phenotype.

\section{Back to the future: the resurgence} of microbial culture collections.

The aforementioned limitations preclude meta approaches to be the one and only tool for plant-microbiome research. In the

\section{BOX 1}

\section{Terminology and definitions.}

Microbiota. Totality of microbes (bacteria, archaea, and fungi) in a particular environment. Refers to taxonomy and abundance of community members.

Amplicon-based community profiling. Phylogenetic marker genes, often of the ribosomal RNA operon (16S or $18 \mathrm{~S}$ genes or the internal transcribed spacer sequence), are utilized to generate DNA amplicon molecules for community profiling. Direct sequencing with second-generation technologies delivers orders of magnitude more sequences compared with sequencing individual vectors after cloning amplicon molecules into clone libraries.

Microbiome. Totality of genomes of a microbiota. Often used to describe the entity of microbial traits (=functions) encoded by a microbiota.

Relationships between organisms. Mutualism refers to a relationship where organisms benefit from each other. Commensalism describes the relationship between two organisms in which one benefits without affecting the other, while in parasitism, one organism benefits at the expense of another. A parasite becomes pathogenic upon causing disease in the host.

Phyllosphere. The microbial habitat defined by the surface of aerial plant organs (dominated by the leaves).

Rhizosphere. The microbial habitat around the root, where the soil's physical, chemical, and biological composition is influenced by the plant. Operationally, this definition can be extended to the root surrounding in nonsoil substrates used for plant growth.

Endosphere. The microbial habitat inside both above- and belowground plant organs.

Plant growth-promoting microbes (PGPM). PGPM encompassing plant growth-promoting rhizobacteria, plant growth-promoting fungi, as well as nonrhizobacterial growth promoters. PGPM are capable of promoting plant growth directly (e.g., through enhanced mineral acquisition from soil) or indirectly (e.g., through enhanced pathogen protection).

Horizontal gene transfer (HGT). HGT refers to the exchange of genes between microorganisms.

Metagenomics. Sequencing is directly applied to a sample comprising a mixture of DNA from various organisms of an environment without (shotgun metagenomics; Riesenfeld et al. 2004) or upon polymerase chain reaction amplification of selected functional genes (gene-targeted metagenomics; Iwai et al. 2010). After bioinformatic analysis, the approach reveals the functional gene complement as well as the phylogenetic composition of organisms (prokaryotes, eukaryotes and viruses) in an environment.

Operational taxonomic units OTU. OTU refer to terminal nodes in a phylogenetic analysis and refer to a group of related sequences (often at $97 \%$ similarity). OTU correspond to community members in amplicon-based microbial profiles.

Agricultural biologicals. Collective term for plantbeneficial products (microbial inoculants, plant extracts, beneficial insects, and others) that can be used by farmers to increase crop health and productivity. 
end, researchers need the ability to manipulate the plant microbiota (varying members in the community) and their inherent traits (varying functional traits in genomes of members) in order to test hypotheses. Therefore, culture collections of genetically tractable microbial isolates represent a valuable tool for increasing our understanding of the plant microbiome. Naturally, this cultivation-based approach also has some inherent limitations, as a central dogma of microbiology affirms that the vast majority of microorganisms on our planet, a figure exceeding $90 \%$, are recalcitrant to in vitro cultivation (Hugenholtz and Pace 1996). However, the number of uncultivable taxa appears lower for members of the plant microbiota, as, for example, for the bacterial community of maize roots an overlap of $48 \%$ was found between bacteria obtained by cultivation and those identified in a clone library (Chelius and Triplett 2001). Furthermore, recent findings and technical developments are bringing microbial collections at the center of the stage in microbiome studies.

It is becoming increasingly clear that the microbial diversity retrieved from sequencing profiles might be largely inflated biases introduced during the amplification and sequencing procedures (Lundberg et al. 2013) as well as during the computational processing of the data (Edgar 2013). Hence, the overlap between the cultured and uncultured microbiota could be greater than previously thought. In a cornerstone manuscript, Goodman and colleagues (2011) reported the development of personalized, indexed bacterial culture collections of the human gut microbiota. In this study, the authors used a dilution approach with a single microbiological medium for large-scale parallel isolation of bacteria inhabiting the human gut microbiota. Subsequently, they determined the taxonomic identity of all retrieved bacterial colonies employing amplicon-based community profiling. Remarkably, this approach demonstrated that approximately $50 \%$ of the bacterial OTU (Box 1) identified through a cultivation-independent survey in human gut samples were cultivable in vitro (Goodman et al. 2011). Not surprisingly, this study spiked the application of similar approaches in plant science. Recently, an investigation compared, in the same forest soil samples, the amplicon-based community profiles with the cultivable fraction of bacteria using eight different microbiological media (VanInsberghe et al. 2013). This study revealed that approximately $22 \%$ of the complex forest soil microbiota is cultivable in vitro. Because of a logistical necessity, the authors utilized a freezing procedure that may have selected against freezing-sensitive but still cultivable bacteria. Therefore, the cultivable fraction from the forest soil microbiota is potentially larger than the observed $22 \%$. Using limiting dilution of Arabidopsis root extracts, a comprehensive culture collection consisting of thousands of bacterial root microbiota members has been established in 96-well microtiter plates (Y. Bai, G. Srinivas, and P. Schulze-Lefert, personal communication). $16 \mathrm{~S}$ ribosomal (r)RNA gene sequencing of these cultures and comparison of the sequences with previous culture-independent $16 \mathrm{~S}$ rRNA gene surveys of the bacterial $A$. thaliana root microbiota revealed that approximately $60 \%$ of rootenriched OTU have corresponding sequence matches in the culture collections. This indicates that a large portion of the $A$. thaliana root microbiota is culturable, although it remains to be tested how many of the cultured microbiota member candidates can recolonize gnotobiotic $A$. thaliana roots under laboratory conditions.

Microbial collections present a highly valuable tool for the development of synthetic microbiota. This will allow testing hypotheses on the molecular bases of host-microbiome interactions under laboratory conditions, as pioneered in medical sciences (Ridaura et al. 2013). In the plant field, Bodenhausen and co-workers (2014) developed an in-vitro system to exam- ine the interaction of Arabidopsis with a synthetic phyllosphere microbiota. In this study, seven bacterial isolates originating from the phyllosphere and representing their most abundant taxa were combined to a synthetic low complexity microbiota and were inoculated on the leaves of axenic Arabidopsis plants. The authors screened a panel of plant mutants and wild accessions of gnotobiotic Arabidopsis and detected reproducible host genotype-dependent perturbations of the inoculated plant microbiota. Of note, this study provided evidence for a direct link between specific host traits and the structural diversification of the microbiota. While this experiment focused on effects on microbiota composition, a similar approach tailored to a synthetic microbiome will offer new opportunities to examine the functional significance in plant-microbiota interactions. However, it is imperative to test whether a similar system can be extended to significantly more complex communities, such as the ones thriving at the root-soil interface, where the plant microbiota is represented by dozens of different microbial taxa (Bulgarelli et al. 2012; Lundberg et al. 2012; Schlaeppi et al. 2014). Furthermore, traditional plant and microbial cultivation substrates, for example, agar-based media, might be useful to acquire biologically meaningful information; nevertheless, plant-microbiome research should also focus on other or additional substrates that better represent soil conditions, such as "transparent soil." Transparent soil refers to a heterogeneous, porous, and synthetic substrate on which plant growth is similar to that in native soil, and this substrate was initially developed for the in situ imaging of living plants and root-associated microorganisms (Downie et al. 2012).

In conclusion, we propose the combination of cultivationdependent and -independent approaches, both with their inherent limitations, for an integral and profound understanding of plant-microbiome interactions. The two types of approaches should become mutually beneficial. For example meta-omics investigations could be helpful to reveal metabolic requirements necessary to cultivate previously unculturable members of the microbiota (Bomar et al. 2011). Likewise, sequencing of representative pure cultures and the genetic and physiological characterization of isolated microbial strains will remain an important task to improve the functional annotation of sequences generated in meta-omics studies (Knief 2014).

\section{Capitalizing on the plant microbiome.}

Feeding mankind relies on high and stable yields from efficient crop production. Modern agriculture is mainly based on the cultivation of high-yield varieties combined with the use of agrochemicals, i.e., fertilizers and chemical products, for nutrient inputs and pathogen control, respectively. Because mineral fertilizers are derived from finite resources and because agrochemicals are often hazardous to the environment, we need alternative and more sustainable agricultural practices (Tilman et al. 2002). Ensuring the sustainability of agriculture becomes more important in light of future challenges such as climate change or the rapid growth of the human population. Taking advantage of the microbiota at work, i.e., the capitalization on microbial traits that are beneficial to the host or the environment or both, presents a promising avenue for the development of a more sustainable next-generation agriculture. In this section, we discuss the translation of basic plant-microbiome research into practice, with the example of exploiting microbial traits to optimize plant growth.

\section{Microbial traits that serve the host plant.}

Mycorrhizal and legume host plants benefit from the microbial provisions of $\mathrm{P}$ and $\mathrm{N}$ by mutualistic mycorrhizal fungi and rhizobia bacteria, respectively (Bouwmeester et al. 2007; Oldroyd et al. 2011). Farmers already take advantage of these 
paradigms of plant-beneficial traits, using mycorrhizal or legume host plants in crop rotations or as cover crops permitting reduction in the use of $\mathrm{N}$ - and P-based fertilizers. However, the root microbiota comprises a multitude of microorganisms other than mycorrhiza and rhizobia with plant-beneficial traits (Lugtenberg and Kamilova 2009; Porras-Alfaro and Bayman 2011). PGPM serve the host plant via either stimulation of plant growth, suppression of plant diseases or pests, or both. PGPM model organisms that are well-studied with regard to their microbial traits and their beneficial effects on plant growth include bacterial genera such as Azospirillum, Bacillus, Pseudomonas, Rhizobium, Serratia, Stenotrophomonas, and Streptomyces, and fungal genera like Ampelomyces, Coniothyrium, and Trichoderma (Berg 2009; de Vrije et al. 2001; Franken 2012).

\section{Exploiting functional traits of microbial inoculants.}

Several of the aforementioned PGPM were commercialized for their application as microbial inoculants (Berg 2009). According to the capitalized microbial traits, these products can be grouped into biofertilizers, plant strengtheners, phytostimulators, and bio-pesticides or biocontrol agents (Lugtenberg and Kamilova 2009). Despite their prospects, many microbial inoc- ulants have not experienced an agronomic break-through with large-scale implementation and, rather, remain a niche product for organic farming. This is partly because microbial inoculants often perform well in controlled laboratory experiments and then do not tap their full potential in natural agricultural settings. One possible explanation is that laboratory experiments are often conducted using sterilized soil substrates, while the microbial inoculants face competition with the native soil microbiota when inoculated to natural soil. It is often unclear if PGPM provide their services to the host plant also in the context of the native soil microbiota.

\section{Targeted application of microbial inoculants.}

Personalized medicine refers to a medical model that proposes to adapt healthcare practices or products tailored to the individual patient. The optimal therapies are chosen based on molecular diagnostics precedent and in the context of the patient's genetic makeup. Inspired by the concept of personalized medicine, next-generation agriculture should aim at customizing the practices and tools (e.g., agricultural biologicals including microbial inoculants [Box 1]) tailored to the individual soil environment. Initial molecular diagnostics could provide the basis for rational choice of appropriate farming treat-

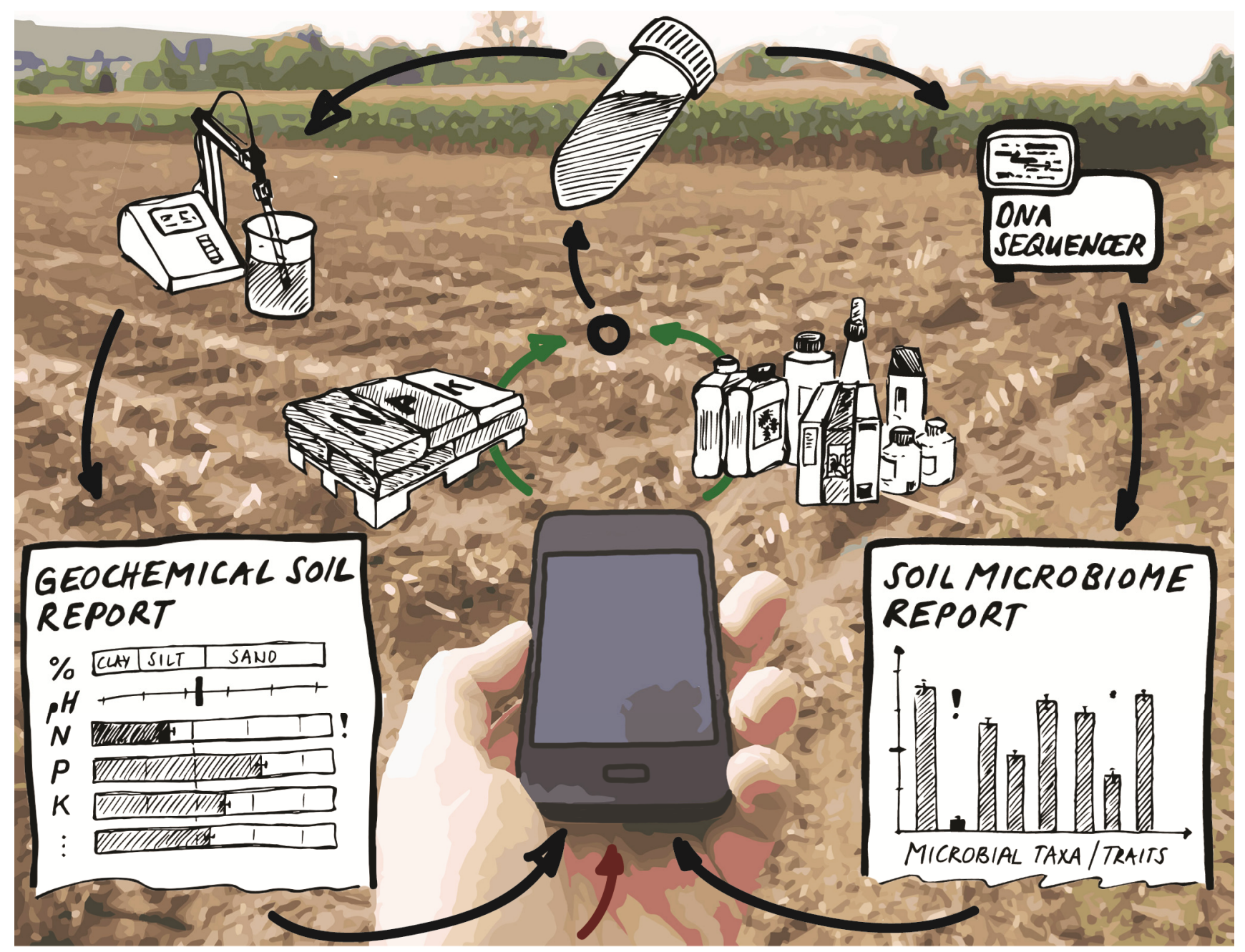

Fig. 1. Towards next-generation agriculture. Field soil samples are analyzed geochemically (left part) and are examined for the soil microbiome employing sequencing technology (right part). The availability of plant nutrients and the occurrence of one or both soil biota and their microbial traits (e.g., $\mathrm{N}$ fixation, phosphorus solubilization, pathogen protection) are reported to the farmer. The smart integration of available geochemical, microbiome, and other relevant information (red arrow) presents the basis for the rational intervention (green arrows) combining field applications of conventional inputs such as mineral fertilizers (left) and agricultural biologicals, including microbial inoculants (right). Additional relevant information includes e.g., the type of plant species (special nutritional requirements?) to be planted or environment factors such as temperature or humidity. The optimization of plant growth based on integrating all involved variates remains largely a black box and requires further research on basic and translational aspects. 
ments. We speculate that considering the genetic context of the local soil microbiome will help to improve and stabilize the effects of microbial inoculants and, therefore, we propose to implement microbiome profiling for a targeted application of microbial inoculants. Figure 1 illustrates an example of translational plant-microbiome research. Today, farmers conduct cost-effective geochemical analyses of their fields by dedicated soil laboratories. The accurate determination of the nutritional status of a field then allows the farmers to apply a targeted supplementation of $\mathrm{N}$ and $\mathrm{P}$ fertilizers. We propose a similar and complementary strategy for the integration of microbial inoculants into farming practices. Imagine a future in which

\section{BOX 2}

Knowledge gaps for translational plantmicrobiome research.

* The application of microbial inoculants can influence, at least temporarily, the native soil microbiota, and we have little information about the buffering capacity of the plant microbiota (Trabelsi and Mhamdi 2013). We need profound knowledge on the survival and persistence of inoculated fungal and bacterial strains in the field and, most importantly, on their effects on the native microbial communities. This is required to understand the biological context-dependency of inoculation approaches and also for the environmental risk assessment of selected natural and, especially, genetically modified microorganisms.

- We also need to understand the abiotic context-dependency of inoculation approaches as, for example, the survival of a microbial inoculum may simply be compromised by inappropriate $\mathrm{pH}$ or temperature conditions. The geochemical composition of the soil is likely an explanatory co-variable for successful inoculations, as some microbial traits might not be beneficial in the plant microbiome under certain conditions. For example, a microbial inoculant with the capacity to solubilize phosphorus might not become established in a soil of high phosphorus availability.

* Microbe-microbe interactions require special attention for the development of approaches combining microbial inoculants, i.e., when a farmer aims to introduce multiple microbial traits in his field. The combination of inoculants may not necessarily produce additive or synergistic effects but could generate a competitive situation with no net benefit.

- Microbes from different taxonomic lineages can harbor the same functional genes and exchange microbial traits within a community by means of HGT. We speculate that the taxonomic identity of a microbial inoculant might be of secondary importance compared with one or more of the actual microbial traits that are introduced to a native microbial community upon inoculation. It remains to be clarified if the success of a microbial inoculant depends on taxonomic community compatibility or, rather, on functional complementary. Is the application of a microbial inoculant effective, when its trait was not previously present in the environment? farmers take soil cores of their fields, send them to dedicated soil laboratories, and contract for soil microbiome analyses based on ever-cheaper sequencing technology. The farmer obtains, for his particular field, a soil microbiome report containing the precise determination of one or both the soil biota and their microbial traits. The soil microbiota can be monitored with amplicon sequencing for community composition or, using shotgun metagenomic sequencing, for the functional gene complement (i.e., the microbial traits). The soil microbiome report then presents the basis for rational intervention with microbial inoculants to the field. The proposed model envisions that farmers optimize their yields by capitalizing on microbial traits and, at the same time, reduce costly and unsustainable inputs of agrochemicals. Although the methods required to quantify the plant microbiome are already available, we believe that the development of the proposed next-generation farming tool requires answering several basic as well as translational questions, which we list as key knowledge gaps in Box 2.

\section{Towards next-generation agriculture.}

The application of microbial inoculants to the field that is rationally based on local microbiome information (Fig. 1) presents one of several conceivable next-generation agricultural tools or practices. Not only the microbial but also the host side of the interaction requires careful attention, since the associated microbiome is emerging as a fundamental plant trait for controlling and optimizing plant growth (Bulgarelli et al. 2013). Therefore, the plant microbiome must become part of future breeding programs so that, for example, next-generation plant cultivars have enhanced capacities to interact with beneficial microbes of the natural soil microbiota or of microbial inoculants. It may be possible to breed for novel crop varieties with improved responsiveness to beneficial microbial traits related to augmented plant nutrient-use efficiency or plant immunity. In the case of controlling pathogen burden, the combined deployment of beneficial services of the plant microbiome (agricultural probiotics) and innate immune functions (resistance genes) is expected to deliver durable and sustainable protection from disease (Dangl et al. 2013). Identification of the genetic components of the host-microbiome control will be key for its ultimate inclusion into breeding programs.

In conclusion, the development of next-generation agricultural tools and practices will depend on the smart integration of all co-variates in the system. The performance of individual plant species and genotype will depend on the microbial inoculants with their specific traits that interact with, one or both, the native soil microbiota and the microbiome under the given climatic conditions in the specific geochemical context of the particular soil environment. Hence, we will exploit the plant microbiome at work and next-generation agriculture will become a reality once we have solved the aforementioned multivariate equation.

\section{ACKNOWLEDGMENTS}

We thank K. Hartman and F. Walder (Agroscope Institute for Sustainability Sciences, Zurich, Switzerland) and Y. Bai (Max Planck Institute for Plant Breeding Research, Cologne, Germany) for assistance and helpful comments on the manuscript. K. Schlaeppi is supported by Agroscope (Institute for Sustainability Sciences, Zurich, Switzerland) and D. Bulgarelli is supported by a Royal Society of Edinburgh/Scottish Government Personal Research Fellowship co-funded by Marie Curie Actions.

\section{LITERATURE CITED}

Bakker, P. A., Berendsen, R. L., Doornbos, R. F., Wintermans, P. C., and Pieterse, C. M. 2013. The rhizosphere revisited: Root microbiomics. Front. Plant Sci. 4:165. 
Berendsen, R. L., Pieterse, C. M., and Bakker, P. A. 2012. The rhizosphere microbiome and plant health. Trends Plant Sci. 17:478-486.

Berg, G. 2009. Plant-microbe interactions promoting plant growth and health: Perspectives for controlled use of microorganisms in agriculture. Appl. Microbiol. Biotechnol. 84:11-18.

Berg, G., Grube, M., Schloter, M., and Smalla, K. 2014. Unraveling the plant microbiome: Looking back and future perspectives. Front. Microbiol. 5:148.

Bodenhausen, N., Bortfeld-Miller, M., Ackermann, M., and Vorholt, J. A 2014. A synthetic community approach reveals plant genotypes affecting the phyllosphere microbiota. PLoS Genet. 10:e1004283. Published online.

Bomar, L., Maltz, M., Colston, S., and Graf, J. 2011. Directed culturing of microorganisms using metatranscriptomics. MBio 2:e0012-00011. Published online.

Bouwmeester, H. J., Roux, C., Lopez-Raez, J. A., and Becard, G. 2007. Rhizosphere communication of plants, parasitic plants and AM fungi. Trends Plant Sci. 12:224-230.

Bulgarelli, D., Schlaeppi, K., Spaepen, S., Ver Loren van Themaat, E., and Schulze-Lefert, P. 2013. Structure and functions of the bacterial microbiota of plants. Annu Rev Plant Biol 64:807-838.

Bulgarelli, D., Rott, M., Schlaeppi, K., Ver Loren van Themaat, E., Ahmadinejad, N., Assenza, F., Rauf, P., Huettel, B., Reinhardt, R., Schmelzer, E., Peplies, J., Gloeckner, F.O., Amann, R., Eickhorst, T., and Schulze-Lefert, P. 2012. Revealing structure and assembly cues for Arabidopsis root-inhabiting bacterial microbiota. Nature 488:91-95.

Chelius, M. K., and Triplett, E. W. 2001. The diversity of archaea and bacteria in association with the roots of Zea mays L. Microb. Ecol. 41:252263

Dangl, J. L., Horvath, D. M., and Staskawicz, B. J. 2013. Pivoting the plant immune system from dissection to deployment. Science 341:746-751.

de Vrije, T., Antoine, N., Buitelaar, R. M., Bruckner, S., Dissevelt, M. Durand, A., Gerlagh, M., Jones, E.E., Luth, P., Oostra, J., Ravensberg, W. J., Renaud, R., Rinzema, A., Weber, F. J., and Whipps, J. M. 2001 The fungal biocontrol agent Coniothyrium minitans: Production by solid-state fermentation, application and marketing. Appl. Microbiol. Biotechnol. 56:58-68.

Delmotte, N., Knief, C., Chaffron, S., Innerebner, G., Roschitzki, B. Schlapbach, R., von Mering, C., and Vorholt, J. A. 2009. Community proteogenomics reveals insights into the physiology of phyllosphere bacteria. Proc. Natl. Acad. Sci. U.S.A. 106:16428-16433.

Downie, H., Holden, N., Otten, W., Spiers, A. J., Valentine, T. A., and Dupuy, L. X. 2012. Transparent soil for imaging the rhizosphere. PLoS One 7:e44276. Published online.

Edgar, R. C. 2013. UPARSE: Highly accurate OTU sequences from microbial amplicon reads. Nat. Methods 10:996-998.

Franken, P. 2012. The plant strengthening root endophyte Piriformospora indica: Potential application and the biology behind. Appl. Microbiol. Biotechnol. 96:1455-1464.

Goodman, A. L., Kallstrom, G., Faith, J. J., Reyes, A., Moore, A., Dantas, G., and Gordon, J. I. 2011. Extensive personal human gut microbiota culture collections characterized and manipulated in gnotobiotic mice. Proc. Natl. Acad. Sci. U.S.A. 108:6252-6257.

Guttman, D., McHardy, A. C., and Schulze-Lefert, P. 2014. Microbial genome-enabled insights into plant-microorganism interactions. Nat. Rev. Genet. 15:797-813

Hugenholtz, P., and Pace, N. R. 1996. Identifying microbial diversity in the natural environment: A molecular phylogenetic approach. Trends Biotechnol. 14:190-197.

Hyung, D., Lee, C., Kim, J. H., Yoo, D., Seo, Y. S., Jeong, S. C., Lee, J. H., Chung, Y., Jung, K. H., Cook, D. R., and Choi, H. K. 2014. Cross-family translational genomics of abiotic stress-responsive genes between Arabidopsis and Medicago truncatula. PLoS One 9:e91721. Published online.

Iwai, S., Chai, B., Sul, W. J., Cole, J. R., Hashsham, S. A., and Tiedje, J. M. 2010. Gene-targeted-metagenomics reveals extensive diversity of aromatic dioxygenase genes in the environment. ISME J. 4:279-285.

Knief, C. 2014. Analysis of plant microbe interactions in the era of next generation sequencing technologies. Front. Plant Sci. 5:216.

Knief, C., Delmotte, N., Chaffron, S., Stark, M., Innerebner, G., Wassmann, R., von Mering, C., and Vorholt, J. A. 2012. Metaproteogenomic analysis of microbial communities in the phyllosphere and rhizosphere of rice. ISME J. 6:1378-1390.

Knight, R., Jansson, J., Field, D., Fierer, N., Desai, N., Fuhrman, J. A. Hugenholtz, P., van der Lelie, D., Meyer, F., Stevens, R., Bailey, M. J., Gordon, J. I., Kowalchuk, G. A., and Gilbert, J. A. 2012. Unlocking the potential of metagenomics through replicated experimental design. Nat. Biotechnol. 30:513-520.

Lebeis, S. L. 2014. The potential for give and take in plant-microbiome relationships. Front. Plant Sci. 5:287.

Lugtenberg, B., and Kamilova, F. 2009. Plant-growth-promoting rhizobacteria. Annu. Rev. Microbiol. 63:541-556.

Lundberg, D. S., Yourstone, S., Mieczkowski, P., Jones, C. D., and Dangl, J. L. 2013. Practical innovations for high-throughput amplicon sequencing. Nat. Methods 10:999-1002.

Lundberg, D. S., Lebeis, S. L., Paredes, S. H., Yourstone, S., Gehring, J. Malfatti, S., Tremblay, J., Engelbrektson, A., Kunin, V., del Rio, T. G., Edgar, R. C., Eickhorst, T., Ley, R. E., Hugenholtz, P., Tringe, S. G., and Dangl, J. L. 2012. Defining the core Arabidopsis thaliana root microbiome. Nature 488:86-90.

Oldroyd, G. E., Murray, J. D., Poole, P. S., and Downie, J. A. 2011. The rules of engagement in the legume-rhizobial symbiosis. Annu. Rev. Genet. 45:119-144.

Philippot, L., Raaijmakers, J. M., Lemanceau, P., and van der Putten, W. H. 2013. Going back to the roots: The microbial ecology of the rhizosphere. Nat. Rev. Microbiol. 11:789-799.

Porras-Alfaro, A., and Bayman, P. 2011. Hidden fungi, emergent properties: Endophytes and microbiomes. Ann. Rev. Phytopathol. 49:291-315.

Ridaura, V. K., Faith, J. J., Rey, F. E., Cheng, J., Duncan, A. E., Kau, A. L. Griffin, N. W., Lombard, V., Henrissat, B., Bain, J. R., Muehlbauer, M. J., Ilkayeva, O., Semenkovich, C. F., Funai, K., Hayashi, D. K., Lyle, B. J., Martini, M. C., Ursell, L. K., Clemente, J. C., Van Treuren, W., Walters, W. A., Knight, R., Newgard, C. B., Heath, A. C., and Gordon, J. I. 2013. Gut microbiota from twins discordant for obesity modulate metabolism in mice. Science 341:1241214.

Riesenfeld, C. S., Schloss, P. D., and Handelsman, J. 2004. Metagenomics: Genomic analysis of microbial communities. Annu. Rev. Genet. 38:525-552.

Schlaeppi, K., van Themaat, E. V. L., Bulgarelli, D., and Schulze-Lefert, P. 2013. Arabidopsis thaliana as model for studies on the bacterial root microbiota. Pages 243-256 in: Molecular Microbial Ecology of the Rhizosphere. F. J. de Bruijn, ed. Kluwer Academic, Dordrecht, The Netherlands.

Schlaeppi, K., Dombrowski, N., Oter, R. G., Ver Loren van Themaat, E. and Schulze-Lefert, P. 2014. Quantitative divergence of the bacterial root microbiota in Arabidopsis thaliana relatives. Proc. Natl. Acad. Sci. U.S.A. 111:585-592.

Sessitsch, A., Hardoim, P., Doring, J., Weilharter, A., Krause, A., Woyke, T., Mitter, B., Hauberg-Lotte, L., Friedrich, F., Rahalkar, M., Hurek, T., Sarkar, A., Bodrossy, L., van Overbeek, L., Brar, D., van Elsas, J. D., and Reinhold-Hurek, B. 2012. Functional characteristics of an endophyte community colonizing rice roots as revealed by metagenomic analysis. Mol Plant Microbe Interact 25:28-36.

Thomas, T., Gilbert, J., and Meyer, F. 2012. Metagenomics-A guide from sampling to data analysis. Microb. Inform. Exp. 2:3.

Tilman, D., Cassman, K. G., Matson, P. A., Naylor, R., and Polasky, S. 2002. Agricultural sustainability and intensive production practices. Nature 418:671-677.

Trabelsi, D., and Mhamdi, R. 2013. Microbial inoculants and their impact on soil microbial communities: A review. Biomed. Res. Int. doi: 10.1155/2013/863240. Published online.

Turner, T. R., James, E. K., and Poole, P. S. 2013. The plant microbiome. Genome Biol 14:209.

VanInsberghe, D., Hartmann, M., Stewart, G. R., and Mohn, W. W. 2013. Isolation of a substantial proportion of forest soil bacterial communities detected via pyrotag sequencing. Appl. Environ. Microbiol. 79:20962098

Vorholt, J. 2012. Microbial life in the phyllosphere. Nature Rev. Microbiol. 10:828-840

Yergeau, E., Sanschagrin, S., Maynard, C., St-Arnaud, M., and Greer, C. W. 2014. Microbial expression profiles in the rhizosphere of willows depend on soil contamination. ISME J. 8:344-358. 